\title{
Association between insulin resistance and left ventricular hypertrophy in sub-saharan hypertensive black patients with preserved ejection fraction: A case control study
}

Bernard KIANU PHANZU ( $\nabla$ doctorkianu@gmail.com )

University Hospital of Kinshasa https://orcid.org/0000-0001-9836-9532

Nkodila Natuhoyila Aliocha

Universite de Kinshasa

Kintoki Vita Eleuthère

University Hospital of Kinshasa

M’Buyamba Kabangu Jean-René

University Hospital of Kinshasa

Longo-Mbenza Benjamin

American School of Kinshasa

\section{Research article}

Keywords: Hyperinsulinemia, insulin resistance, obesity, sedentary sitting time, left ventricular hypertrophy, left ventricular mass, hypertension, Black, sub-saharan African

Posted Date: June 11th, 2020

DOI: https://doi.org/10.21203/rs.3.rs-33818/v1

License: (c) (i) This work is licensed under a Creative Commons Attribution 4.0 International License. Read Full License

Version of Record: A version of this preprint was published on January 2nd, 2021. See the published version at https://doi.org/10.1186/s12872-020-01829-y. 


\section{Abstract}

\section{Background:}

Conflicting information exists regarding the association between insulin resistance (IR) and left ventricular hypertrophy (LVH).

We described the associations between parameters of obesity, fasting insulinemia, HOMAIR with LVH in black patients with essential hypertension.

\section{Materials and Methods:}

A case-control study was conducted at the Centre Médical de Kinshasa (CMK), the Democratic Republic of the Congo, between January and December 2019. Cases and controls were hypertensive patients with and without LVH, respectively. The relationship between obesity indices, physical inactivity, parameters of glucose metabolisme and lipid disorders and LVH were assessed using linear and logistic regression analyses in simple and univariate exploratory analysis, respectively. When differences were observed between LVH and the independent variables, the effect of potential confounders was studied by adjustment in multiple linear regression and in conditional logistic regression in multivariate analysis. The coefficients of determinations $\left(\mathrm{R}^{2}\right)$, the adjusted ORs and their $95 \% \mathrm{Cl}$ were calculated to determine the association between the LVH and the independent variables

\section{Results:}

Eighty-eight cases (52 men) were compared to 132 controls (81 men). Nineteen percent of left ventricular mass (LVM) variation (19\%) was predicted by age, $31.3 \%$ by the duration of hypertension, $44.4 \%$ by BMI, $42.5 \%$ by WC, $20 \%$ by glycemia, $44.8 \%$ by insulinemia and $43.7 \%$ by HOMAIR. In multiple linear regression analysis, duration of hypertension, Body Mass Index (BMI), insulinemia and HOMAIR explained $68.3 \%$ of the variability in the increase in LVM. In the logisitic model obesity multiplied the risk of LVH by 3 (aOR: $2.8,95 \% \mathrm{Cl}(1.06-7.4), \mathrm{p}=0.038)$, IR by 8 (aOR: 8.4, 95: (3.7-15.7), $\mathrm{p}<0.001)$.

\section{Conclusion:}

Obesity and IR appear to be the main predictors of LVH. The comprehensive management of cardiovascular risk factors should be emphasized with particular attention to obesity and insulin resistance. A prospective black sub-saharan population based study with serial imaging remain essential to better understand subclinical LV deterioration over time and to confirm the role of insulin resistance in black sub-saharan hypertensives.

\section{Background}

Hypertension is a major contributor to global mortality and incapacity [1]. In 2018, the World Health Organization (WHO) listed hypertension as one of the two main global risk factors of death and disability 
for all ages, alongside smoking [2]. The number of people with systolic blood pressure (SBP) of $140 \mathrm{~mm}$ $\mathrm{Hg}$ or higher was estimated to have increased from 442 million in 1990 to 874 million in 2015, when this SBP level was responsible for $14 \%$ of total deaths and 143 million disability-associated life-years [3]

Hypertension is a major challenge, especially for sub-Saharan African countries where its prevalence, which was $30.0 \%$ according to a pooled data from 33 surveys published between 2000 and 2013[4], is experiencing dramatic growth. Hypertention in sub-Saharan Africa affects younger people, is poorly controlled and often leads to target organ damage at an earlier age and with more severe cardiovascular complications $[2,5]$.

Hypertensive patients with insulin resistance (IR) are at increased risk of cardiovascular events compared to hypertensive patients without IR [6]. Similarly, the presence of target organ damage (TOD), including LVH has a negative prognostic value in hypertensive patients $[7,8]$. The European Guidelines on the management of hypertension recommend that hypertensive patients with a target organ, including LVH, be considered at a higher risk of cardiovascular events [9].

The high prevalence of IR in the hypertensive population was first highlighted in 1966 by Welborn et al. from a sample of 19 non-diabetic hypertensives [10]. Two decades later, this finding was confirmed from the results of other research studies with more participants. Ferrannini in 1987 was the first researcher to state hypertension was a state of insulin resistance [11].

In 1997, the existence of a link between IR and hypertension was definitively confirmed by the European Group for the Study of Insulin Resistance (EGIR) which demonstrated that regardless of age, gender or body mass index, the level of blood pressure is positively correlated with insulin resistance and insulinemia [12].

Gerald M. Reaven, fondly remembered as the father of IR because of his contribution to understanding the central role of IR in cardiovascular disease, developed the insulin suppression test; the first quantitative method to assess insulin-mediated glucose uptake in humans [13]. Using this test, he established the importance of IR in human disease, and especially in type 2 diabetes $[14,15]$. In a nondiabetic patient population, he illustrated the role of insulin resistance in the development of, essential hypertension [16], the osmotic balance [17], stimulation of the sympathetic nervous system [18], hypercoagulability [19], decreased clearance of urinary uric acid with resultant hyperuricemia [20], increased postprandial lipemia and accumulation of residual lipoproteins [19] and occurrence of lipid abnormalities such as hypertriglyceridemia [21], low HDL-c [22], and a decrease in the diameter of LDL-c particles [23].

IR also plays a key role in the development of left ventricular remodeling (LVR) [24, 25]. LVH, one of the phenotypes of LVR linked to hypertension, has a poor prognostic value $[7,26,27]$. Both mechanical and hemodynamic factors as well as the trophic role of insulin in hypertension favor the occurrence of LVH [28]. Genetic factors as well as environmental factors also play their role [29-31] in the development of 
LVH and its resultant consequences. The link between echographic LVH and cardiovascular risk has been largely documented in the literature [26, 32-37].

There exists, however, conflicting information regarding the association between insulin resistance and left ventricular hypertrophy in hypertensive patients. We sought to assess such relationship among a hypertensive sub-saharan black population

\section{Material And Methods}

\section{Study design and setting}

This is a case-control study conducted at the Centre Médical de Kinshasa (CMK) between January and December 2019. The CMK is a reference clinic, working on international standards and norms, with a cardiology unit named "pôle de cardiologie » with highly qualified and regularly retrained personnel, that provides cardiovascular explorations such as doppler echocardiography, coronary scanner and cardiopulmonary exercise testing. A cardiovascular rehabilitation unit, the only one in central Africa, is also operational there.

\section{Patient selection}

Consecutive asymptomatic patients with probably essential hypertension aged 20 years or more attending the outpatient clinic at the CMK Pôle de cardiologie between January and December 2019 were screened for clinical or laboratory evidence of secondary hypertension, renal or hepatic disease. They were invited by writen informed consent to participate in the present study. Those with high blood pressure unrelated heart disease were not enrolled. Each participant who met the criteria for echocardiographic diagnosis of LVH was matched for gender and age with two hypertensive patiens without left ventricular hypertrophy.

A total of 267 participants were screened, 106 with left ventricular hypertrophy and 161 without left ventricular hypertrophy. Of these, 47 were excluded due to dilated cardiomyopathy in 20 participants (8 with LVH and 12 without LVH), ischemic cardiopathy in 14 participants ( 5 with LVH and 9 without LVH), significant valvulopathy in 5 participants ( 2 with LVH and 3 without LVH), pericardidis in 5 participants without LVH, and hypertrophic cardiomyopathy in 3 participants with LVH. The final analysis therefore included 220 participants 88 (40\%) with and 132 (60\%) without left ventricular hypertrophy.

\section{Study procedures}

\section{Anamnestic data}

Demographic data (age, sex), lifestyle habits (heavy alcohol consumption, current smoking, sedentary behavior), medical history including cardiovascular risk factors (age at diagnosis of high blood pressure, history of diabetes mellitus, dyslipidemia, hyperuricemia, menopause) and previous cardiovascular events (stroke, ischemic heart disease, heart failure, Chronic Kidney Disease, cardiovascular surgery), and 
current medication use for chronic disease (antihypertensive treatment, anti-diabetic treatment and other treatments including statins, antiplatelet agents, hypouricemics, oral contraception, hormone replacement therapy) were collected during an in-person directed interview using ad hoc questionnaire.

\section{Anthropometric data}

Anthropometric parameters were measured by a final year medical student who had also undergone a study-training session held by the authors. The student measured both primary variables (weight, height, waist size, hip measurement) according to WHO recommendations and a derived variable (body mass index (BMI) as :

- Body weight was measured to the nearest $100 \mathrm{~g}$ using a validated electronic balance the participants upright in light clothing without shoes ;

- The height was obtained to the nearest centimeter using a measuring rod, , with the participant standing, barefoot and bareheaded;

- The waist circumference to the nearest $0.1 \mathrm{~cm}$, was mesured using a measuring tape applied directly to the skin along a horizontal line passing through the umbilicus.

- The body surface area (BSA) was calculated using the DuBois formula [38] as follows: BSA = Height $0.725 \times$ Weight $0.425 \times 0.007184$.

- BMI was obtained by dividing the weight $(\mathrm{Kg})$ by the square of height $\left(\mathrm{m}^{2}\right)$

\section{Blood pressure}

BP was measured non-invasively by 24 hour-ambulatory blood pressure monitoring (ABPM) using a TONOPORT V (GE Health care, Freiburg, GERMANY) type recorder. During this recording, the participant was asked to maintain his usual way of life.

\section{Echocardiographic data}

Left ventricular measurements were taken according to the 2015 American Society of Echocardiography and the European Association of Cardiovascular Imaging updated guidelines for cardiac chamber quantification [39] using a Vivid T8 (GE) type ultrasound system equipped with $3.5 \mathrm{MHz}$ transducers. Two-dimensional guided M-mode echocardiography was performed on a parasternal long-axis view. Interventricular septum (IVS) thickness in diastole (IVSd), left ventricular posterior wall (PW) thickness in diastole (LVPWd), and left ventricular end-diastolic diameter (LVEDd), all measured in mm were assessed at a level just below the mitral valve leaflets at end-diastole. Simultaneous ECG was done to correlate left ventricular measurements with the cardiac cycle. Diastolic wall thickness was measured at the onset of the QRS wave. LVM was calculated based on the American Society of Echocardiography simplified cubed equation linear method using the following equation : $\operatorname{LVM}($ grams $)=0.8 \times 1.04 \times[($ LVEDd + IVSd + LVPWd $\left.)^{3}-(\text { LVEDd })^{3}\right]+0.6 \mathrm{~g}$. LVM was indexed to BSA and to height as mass/BSA and mass/height ${ }^{2.7}$. The relative wall thickness (RWT) of the left ventricle (LV) was calculated as follows: $(2 \times$ LVPWd) / 
LVEDd. In accordance with international recommendations [40], the parameters of LV diastolic function were measured by recording transmitral flow velocity using conventional doppler echocardiography. With pulsed wave Doppler (PW), transmitral flow velocity was recorded from the apical transducer position with the sample volume situated between the mitral leaflet tips. E (Peak E-wave velocity) and A (Peak Awave velocity) and Deceleration time of early filling (DT), were recorded in apical four-chamber with color flow imaging for optimal alignment of PW Doppler with blood flow. PW Doppler sample volume (1-3 mm axial size) was placed between mitral leaflet tips, using low wall filter setting (100-200 MHz) and low signal gain, so that the optimal spectral waveforms should not display spikesand. Both $E, A$ and DT were measured as the averages of five consecutive cardiac cycles The E/A ratio was calculated. Tissue Doppler echocardiography, which measures the velocity of the regional cardiac wall, was performed by activating the tissue doppler echocardiographic function, as for two dimensional and M-mode echocardiography. Mitral annular velocities were recorded from the apical window. Sample volumes were located at the lateral site of the mitral annulus. Peak early diastolic mitral annular velocity $\left(E^{\prime}, \mathrm{cm} / \mathrm{s}\right)$ was measured over five cardiac cycles and the mean calculated. The ratio $\mathrm{E} / \mathrm{e}^{\prime}$ was used as a parameter of left atrial pressure, which is elevated with progression of LV diastolic dysfunction. These parameters, obtained by tissue doppler echocardiography, were also used as parameters of LV diastolic function.

\section{Laboratory measurments}

For all analyses, a blood sample was taken from the cubital vein between 7 a.m. and 9 a.m. following an overnight fast that started at $10 \mathrm{pm}$ the previous day. All analyses were carried out at the CMK laboratory. Blood was collected in a dry tube for the assessment of serum uric acid level, total cholesterol, LDLcholesterol, HDL-cholesterol and triglycerides,. Serum was used for the analysis. The assay was performed by the standard colorimetric method. Readings were measured using the colorimetric spectrophotometer brand HELIOS Epsilon (Milwaukee, USA). The blood glucose test was performed on plasma oxalate by colorimetric method using standard reagents Biolabo.

The insulin dose was assessed on EDTA plasma by ELISA. Ooptical density reading was done on a string read from the firm HUMAREADER HUMAN (Germany).

Assessments of glycated hemoglobin were performed on plasma treated with EDTA by the electrophoretic method using HYRYS HYDRASIS from the firm SEBIA (France).

Serum creatinine was measuredby the method of simple colorimetric Jaffe. Readings were assessed by colorimetric spectrophotometer (Spectrum 2100 brand, South Africa).

\section{Operational Definitions}

\section{Lifestyle data}

Sedentary was defined as sitting for more than 7 hours a day [41]. 
Cigarette smoking was defined as regular smoking for at least 30 days preceding the interview date regardless of the number of cigarettes smoked [42].

Excessive alcohol consumption was defined as drinking more than 2 glasses of beer or its equivalent every day for at least a year [43].

\section{Anthropometric parameters}

Overweight was defined as a BMI between 25 and $29.9 \mathrm{Kg} / \mathrm{m} 2$ of body surface area [44].

Obesity was defined as a BMl equal to or greater than $30 \mathrm{Kg} / \mathrm{m} 2$ of body surface area [44]. Abdominal obesity was been defined as a waist circumference of more than $>102 \mathrm{~cm}$ and $>88 \mathrm{~cm}$ for men and women respectively [44].

\section{Bioclinical data}

Poor control of arterial hypertension was defined as an average systolic blood pressure greater than 130 $\mathrm{mmHg}$ and/or average diastolic BP greater than $80 \mathrm{mmHg}$ on 24-hour ambulatory blood pressure monitoring [45].

\section{Paraclinical data}

Diabetes mellitus was defined as a fasting blood glucose $\geq 10 \mathrm{mmol} / \mathrm{I}$ with a glycated hemoglobin level greater than $7 \%[46]$.

Hyperinsulinemia was been defined as fasting insulin $>90 \mathrm{mmol} / \mathrm{L}$.

Insulin resistance was defined as a HOMAIR of $\geq 2.5$ [47].

Dyslipidemia was defined as a HDL-cholesterol level of $<1.03 \mathrm{mmol} / \mathrm{L}$ for males or $<1.04 \mathrm{mmol} / \mathrm{L}$ for females, and/or an LDL-cholesterol level $\geq 3.38 \mathrm{mmol} / \mathrm{L}$, and/or a total cholesterol level $\geq 5.17 \mathrm{mmol} / \mathrm{L}$, and/or a triglyceride level $\geq 1.69 \mathrm{mmol} / \mathrm{L}$ [48].

The atherogenicity index (AI) was calculated by the total cholesterol to HDL-C ratio. The atherogenicity index was considered high when this ratio was greater than 5 [49].

Hyperuricemia was defined as a uric acid of $>420 \mathrm{mmol} / \mathrm{L}[50]$.

\section{Echographic data}

Normal LVM was defined as $\leq 115 \mathrm{~g} / \mathrm{m} 2$ or $\leq 48 \mathrm{~g} / \mathrm{m} 2.7$ for males and $\leq 95 \mathrm{~g} / \mathrm{m} 2$ or $\leq 44 \mathrm{~g} / \mathrm{m} 2.7$ for females; LVH was defined as LVM exceeding these values in males and female patients respectively. Four LV geometric patterns were defined as follows [51, 52] : normal geometry (normal LVM and RWT $\leq$ 0.42 ), concentric remodeling (normal LVM and RWT $>0.42$ ), eccentric hypertrophy (LVH and RWT $\leq 0.42$ ) and concentric hypertrophy (LVH and RWT > 0.42). 
Three patterns of diastolic dysfunction (DD) were defined as follows [53, 54]: abnormal relaxation (grade I of DD: E/A ratio < 1 and prolonged deceleration time), pseudonormal relaxation (grade II: E/A ratio $>1$ and intermediate values of deceleration time), and restrictive patterns (reversible and irreversible, grade III-IV respectively; E/A ratio $>2$ and shortened deceleration time).

The dilation of the left atrium was defined as an area of the $O G$ of $>20 \mathrm{~cm} 2$ of body surface [39].

\section{Statistical Analyses}

Data were presented in the form of absolute (n) and relative (\%) frequencies for categorical variables and as averages ( \pm standard deviation) for quantitative variables. Paired comparisons between the cases and controls were made using Pearson square Chi-square test or the Fisher Exact test as appropriate for categorical variables and using Student's t-test for continuous variables.

Linear regression was used to determine factors predictive of LVM variations. The following variables were entered in the univariate analysis : parameters of obesity (WC, $\mathrm{HC}, \mathrm{BMI})$, parameters of glucose metabolism (Fasting glucose, HBA1c, fasting insulinemia, and HOMAIR), parameters of lipid metabolism (TC, HDL-C, LDL-c, Triglycerides), parameters of renal function (creatinine and uricemia), parameters of phosphocalcic metabolism (calcium, ionized calcium, phosphore). When significant associations were observed between LVM and the independent variables, the effect of potential confounders was studied by adjustment in multiple linear regression.

Simple logistic regression was used to determine factors predictive of LVH. The following variables were entered in the univariate analysis : Medical \& social history (Known HTN, ND HTN, Cigaret smoking, excessive alcohol consumption, Menopausal), sedentary, uncontrolled HTN, dislipidemia, High Al, diabetes mellitus, hyperinsulinemia, hyperuricemia and insulin resistance. When associations were observed between LVH and the independent variables, the effect of potential confounders was studied by adjustment in conditional logistic regression (multivariate analysis).

The significance threshold retained was then $p<0.05$. Statistical analyzes were performed using XLStat 2020 and SPSS (Statistic Package for Social Sciences) for Windows version 24 software

\section{Ethical considerations}

This research was conducted in strict compliance with the recommendations of the Helsinki Declaration III. Approval to conduct the study was obtained from the ethics committee of the University of Kinshasa School of Public health. Each participant provided written informed consent for to participate in the study. All respondents were debriefed on the results of the study.

\section{Results}

\section{Characteristics of cases and controls}


Compared to patients without LVH, patients with LVH had significantly higher ( $\mathrm{p} \otimes 0.05) \mathrm{BMI}, \mathrm{WC}, \mathrm{HC}$ and average 24-hour systolic blood pressure. There was a significantly higher proportion of sedentary persons among patients with LVH (Table 1, at the end of the document text file) with signficanlty higher relative wall thickness, E-wave deceleration time, triglyceridemia, Al, glycemia, HbA1c,insulinemia, HOMAIR, IR and hyperuricemia (Table 2, at the end of the document text file).Conversely, HDL-c level and E/A ratio were signficantly lower in patients with LVH. Cases and controls did not differ significantly with respect to the matching variables (Table 1 and 2 )

\section{Determinants of Left Ventricular mass}

In Simple linear regression, as illustrated in Table 3 (at the end of the document text file), there was a significant and positive relationship between LVM and age, duration of hypertension, BMI, WC, Glycemia, insulinemia and HOMAIR.

Nineteen of LVM (19\%) was predicted by age, $31.3 \%$ by the duration of hypertension, $44.4 \%$ by $\mathrm{BMI}, 42.5 \%$ by WC, $20 \%$ by glycemia, $44.8 \%$ by insulinemia and $43.7 \%$ by HOMAIR (Figure $1 \mathrm{~A}$ and Figure $1 \mathrm{~B}$, at the end of the document text file).

In multiple linear regression, patients predicted LVM is equal to 0.56 (hypertention duration) +0.67 (BMI) + 0.08 (Insulin levels) +0.27 (HOMAIR).

The duration of hypertension, BMI, insulin and HOMAIR predicted $68.3 \%$ of the patients LVM (Table 4 , at the end of the document text file).

\section{Determinants of LVH}

In univariate analysis, global obesity, abdominal obesity, sedentary, atherogenicity index, hyperuricemia, IR were signficants predicators of LVH.

After multivariate adjustment, only total obesity and IR persisted as independent determinants of LVH. Obesity increased the risk of LVH three-fold (OR 2.8, 95\% Cl 1.06-7.40, $p=0.038)$ and IR increased it eightfold (OR 8.4, 95\% Cl3.7-15.7, $\mathrm{p}<0.001$ ) (Table 5, at the end of the document text file).

\section{Discussion}

In this study, four factors which explained the bulk of the increase in LVM, (68\% of it), were established. These were the duration of hypertension, BMI, insulinemia and HOMAIR. However, only insulin resistance and total obesity emerged as the independent determinants of LVH. We also observed that patients with LVH were more often sedentary, had higher obesity parameters, and more abnormalities in carbohydrate and lipid metabolism compared to patients without LVH. In addition, they also had significantly higher uric acid levels and atherogenicity index, as well as a lower E/A ratio and a longer mitral E wave deceleration time. 
Conflicting information exists regarding the involvement of insulin resistance in the development of LVH. Costa et al (1995) did not find any relationship between IR (with insulin measured during glucose tolerence test) and LVM in a small sample of 35 non-obese hypertensive Brazilians [55]. Galvan et al (2000), after adjusting for blood pressure and body mass index (BMI), also found that IR (With insulin sensitivity measured by the insulin clamp technique) was not an independent determinant of LVH in a small sample of 50 Italian nondiabetic subjects[56]. These results are opposite to those found in the present study. The difference in profile of the study population, the sample size and the methods used to diagnose insulin resistance could explain this difference. In our study, HOMAIR was used to diagnose IR. This method has the advantage of a simpler implementation than the hyperinsulinemic euglycemic glucose clamp wich is the gold standard method for the determination of insulin sensitivity[57]. HOMAIR has been the subject of numerous validations which have shown a satisfactory correlation with the gold standard method ( $r=0.72$ to 0.82 depending on the studies) with no notable difference according to sex, age, weight, diabetic or hypertensive status [58]. Our results concurr however, with data obtained in populations other than black sub-Saharan africans. Sasson et al. (1993) demonstrated a significant association between IR and LVH which was independent of blood pressure level and BMI [59]. Lind et al (1995) also found such an association and demonstrated that hyperinsulinemia was responsible for $43 \%$ variation in the left ventricular mass [60]. In a recent prospective population study, Cauwenberghs et al (2018) found that basal insulin resistance / hyperinsulinemia and its worsening during follow-up predicted left ventricular remodelling [61].

The pathophysiological arguments that can support this association are as follows: It is now recognized that LVH is mediated not only by mechanical stress from pressure overload, but also by various neurohormonal substances and metabolic abnormalities that independently exert trophic effects on cardiomyocytes and the extracellular matrix $[28,62]$. This is substantiated by the high prevalence of LVH in normotensive type 2 diabetics $[63,64]$. Insulin resistance, by multiple and complex mechanisms, has been shown to promote hypertrophy of cardiomyocytes and matrix deposition, regardless of its effect on systemic blood pressure [65].

The transmembrane transport and mitochondrial oxidation of glucose are reduced due to a down regulation of the expression of glucose transporter-4 in response to insulin resistance [66]. Therefore energy metabolism then depends on the oxidation of fatty acids for more than $90 \%$ of its needs leading to an increase in plasma levels of fatty acids. The predominant oxidation of fatty acids and reduction in the energy supply from glucose and pyruvates, lead to the formation of end products of non-enzymatic glycation (AGE Advanced Glycation End products), an excess of glycolytic compounds and increased synthesis of ceramide, all of which promote apoptosis. AGE bind to specific receptors, activate a Protein Kinase $\mathrm{C}$ whose overexpression stimulates the growth factor of median connective tissue, synthesis of collagen and interstitial fibrosis. Additionally, insulin resistance and an increase in the mitochondrial influx of fatty acids predispose to an overproduction of superoxide ions involved in the genesis of hypertrophy, fibrosis, and left ventricular dysfunction. Furthermore, IR generates reactive oxygen species which are involved in the genesis of LVH and fibrosis [67]. 
The association between the duration of hypertension and LVH has been highlighted in several previous studies. In the Democratic Republic of Congo, Lepira et al have shown that the duration of hypertension predicted the occurrence of electrical LVH [68]. This association accounts for the influence of the duration of myocardial exposure to chronic barometric overload represented by hypertension.

In the present study we found that hypertensive subjects with LVH had a lower E/A ratio and a longer deceleration time that indicate abnormality in relaxation [52,54]. The diastolic dysfunction is a consequence of both IR $[69,70]$ and left ventricular hypertrophy, and underlying myocardial fibrosis [54, 71-74]. In addition, a mitochondrial dysfunction which accompanies insulin resistance state is thought to play a role in both left ventricular hypertrophy and diastolic dysfunction [75]. However, this is still a subject of debate. On the one hand, a certain degree of diastolic dysfunction exists in hypertensive patients long before they develop LVH [76], on the other hand, regression of LVH after antihypertensive treatment does not necessarily lead to the normalization of diastolic function [77]. Nevertheless, some studies found that normalization of the left ventricular mass leads to normalization of diastolic function [78].

Our hypertensive patients with LVH were often sedentary, with higher obesity parameters and more abnormalities in carbohydrate and lipid metabolism. Controversies exist regarding the relationship between sedentarity and left ventricular mass. Gibbs et al. (2014) observed relationships between sedentary lifestyle and obesity, and higher LVM in Caucasian adults, but not in black populations [79]. In a previous analysis we assessed this association in a population of sub-Saharan blacks and Maghrebis of white skin population, and found that sedentary lifestyle was associated with a lower left ventricular mass among white Maghrebian population but not in sub-Saharan blacks [80]. Likewise, in the present study of sub-Saharan blacks, although a larger proportion of patients with LVH were sedentary, no significant association was found between sedentary lifestyle and left ventricular mass. It is possible that a potential qualitative difference exists in the cardiovascular consequences of sedentary.

The association between obesity and left ventricular hypertrophy appears to be a common finding. However, there exists a divergence as regards the concentric or eccentric geometry pattern of this hypertrophy in obese hypertensive patients. Some authors have found a predominance of eccentric geometry [81] whereas others, including ourselves, have found a predominance of concentric geometry $[82,83]$. Concentric geometry is more often attributed to pressure overload whereas eccentric geometry is attributed to volume overload [84]. When hypertension, a condition of pressure overload and obesity, wich is a condition of volume overload, coexist, the resulting hypertrophic phenotype would be determined by the predominance of one over the other. This may explain the diverging results in the literature based on the study population. Furthermore, an initially concentric geometry can evolve over time towards an eccentric geometry.

The sedentary-obesity couple is essentially characterized by a chronic caloric excess. Experimental research has shown that prolonged and uninterrupted sitting sessions lead to an increase in blood levels of insulin and glucose. Obesity is linked to insulin resistance via complex mechanisms e.g., inflammation 
due to the accumulation of lipids, the inhibitory effect of fatty acid oxidation on glucose oxidation, and the secretion of adipocytokines which have all been associated with the development of local and systemic insulin resistance [85]. Therefore, insulin resistance would bridge the gap between between sedentary lifestyle / obesity and left ventricular hypertrophy.

Significantly higher uric acid levels were found in hypertensives with LVH when compared to hypertensives without LVH. This is in agreement with previous studies which have shown that hypertensives with LVH have higher uric acid levels [86-90]. A large prospective population-based study in Italy found that hyperuricemia is a predictor of LVH [91]. A causal link was suggested since normalization of uric acid levels using hypouricemic treatment led to a a reduction in the left ventricular mass [92, 93]. Several mechanisms could be used to explain the increase in LV mass due to hyperuricemia, these includ the systemic inflammatory response, oxidative stress [94, 95], activity of the renin system angiotensin aldosterone [96], endothelial dysfunction [97] and the expression of endothelin-1 in cardiac fibroblasts that promotes interstitial fibrosis in myocardium [98]. Furthermore, some indirect effects of hyperuricemia, such as increased BP, parallel decrease in glomerular filtration rate (GFR), deterioration in adhesion and platelet aggregation, and increased aortic stiffness, could further contribute to the development of LVH [99].

Finally, this study found a higher atherogenicity index in hypertensives with left ventricular hypertrophy compared to hypertensives without left ventricular hypertrophy, which suggests an increase risk of coronary events. This aligns to previous study which established that LVH is a risk factor for coronary heart disease mortality [100].

\section{Study Limitations}

Our study has to be interpreted within the context of its potential limitations and strengths. First, echocardiographic measurements are prone to measurement errors as a result of signal noise, acoustic artefacts, and angle dependency. We howerver had an experienced cardiologist with post-gradued training in cardiac imaging perform all echocardiograms. Secondly, the case-control design we used meant we could not realy assess cause-effect relationships. Third, the in-hospital and monocentric desing makes it risky to extrapolate the results to all sub-Saharan black hypertensives. our study covers a gap, to the extent that, to the best of our knowledge. This is, to the best of our knwowledge, the first description of the association between IR and LVH in sub-saharan hypertensive black patients.

\section{Conclusions}

Our results showed direct and significant associations between the duration of hypertension, the body mass index, insulinemia and HOMAIR and left ventricular mass. Insulin resistance and obesity emerged as independent determinants of left ventricular hypertrophy in hypertension. We recommend concomitant management of associated cardiovascular risk factors when managing patients with hypertension with particular attention paid to insulin resistance and obesity. A prospective black sub-saharan population 
based study with serial imaging remain essential to better understand subclinical LV deterioration over time and to confirm the role of insulin resistance in black sub-saharan hypertensives.

\section{List Of Abbreviations}

$\mathrm{ABPM}=$ ambulatory blood pressure monitoring

$\mathrm{Al}=$ atherogenicity index

$\mathrm{BMI}=$ Body mass index

BSA = Body Surface Area

DBP=Diastolic Blood Pressure.

$\mathrm{DT}=$ deceleration time

$E / A=$ ratio of peak early and late diastolic flow velocities

$\mathrm{E}=$ mitral $\mathrm{E}$ wave

$\mathrm{HbA} 1 \mathrm{C}=$ glycated haemoglobin

$\mathrm{HC}=$ hip circumference

HDLC=high density lopoprotein

HOMAIR=Homeostatic Model Assessment for Insulin Resistance

$\mathrm{HR}=$ Heart rate

HTN=hypertension

$\mathrm{IR}=$ insulin resistance.

IVS=interventricular septal thickness

$\mathrm{LAA}=$ left atrium area

LDLC=low density lipoprotein

LDL-C=low density lipoprotein

LVED=Left ventricular end-diastolic diameter

$L V E F=$ Left ventricular ejection fraction 
LVM=left ventricular mass

LVMbs=left ventricula mass indexed to body surface area

LVMIh=left ventricular mass indexed to hight 2,7

ND HTN=newly-diagnosed hypertension

PWT=Posterior wall thickness

RWT=relative wall thickness

SBP=Systolic Blood Pressure

SPAP=systolic pulmonary arterial pressure

SWT=Sum of wall thickness

T2DM=type 2 diabetes melitus

$\mathrm{TC}=$ total cholesterol

WC=Waist circumference

\section{Declarations}

Ethics approval and consent to participate: Ethics approval and consent to participate the study was approved by the research ethics committee at Public health school of Kinshasa. All participants provided informed consent.

Consent for publication: Not applicable.

Availability of data and materials: Because consent given by study participants did not include data sharing with third parties, anonymized data can be made available to investigators for analysis on reasonable request to the corresponding author.

\section{Competing interests}

The authors had no conflicts of interest to declare in relation to this article.

Funding: None.

\section{Authors' contributions}

Design and concept of study: Kianu Phanzu Bernard 
Acquisition of data: Kianu Phanzu Bernard

Manuscripti draft: Kianu Phanzu Bernard

Supervision: Kintoki Vita Eleuthère, Longo-Mbenza Benjamin, M’Buyamba-Kabangu Jean-René

Statistical analysis: Nkodila Natuhoyila Aliocha

All authors read and approved the final manuscript.

\section{Acknowledgements}

We would gratefully acknowledge Dr Rodolph Amhed, Managing Director of the Centre Médic de Kinshasa for granting us permission to conduct this study in CMK;

\section{References}

1. Huffman MD, Lloyd-Jones DM. Global burden of raised blood pressure: coming into focus. JAMA. 2017;317(2):142-3.

2. GBD 2017 Mortality Collaborators. Global, regional, and national age-sex-specific mortality and life expectancy, 1950-2017: a systematic analysis for the Global Burden of Disease Study 2017. Lancet. 2018;392(10159):1684-1735.

3. Forouzanfar, M.H., et al., Global burden of hypertension and systolic blood pressure of at least 110 to 115 mm Hg, 1990-2015. JAMA, 2017. 317(2): p. 165-182.

4. Ataklte, F., et al., Burden of undiagnosed hypertension in sub-saharan Africa: a systematic review and meta-analysis. Hypertension, 2015. 65(2): p. 291-8.

5. Mittal BV, Singh AK. Hypertension in the developing world: challenges and opportunities. Am J Kidney Dis, 2010. 55(3): p. 590-8.

6. Bigazzi, R., et al., Increased cardiovascular events in hypertensive patients with insulin resistance: a 13-year follow-up. Nutr Metab Cardiovasc Dis, 2008. 18(4): p. 314-9.

7. Castello Brescane, R., The prognostic significance of left ventricular geometry: fantasy or reality? Rev Esp Cardiol, 2009. 62(3): p. 235-8.

8. Barsukov, A.V., et al., [Left ventricular hypertrophy as a marker of adverse cardiovascular risk in persons of various age groups]. Adv Gerontol, 2014. 27(4): p. 763-70.

9. Williams, B., et al., $2018 \mathrm{ESC} / \mathrm{ESH}$ Guidelines for the management of arterial hypertension. Eur Heart J, 2018. 39(33): p. 3021-3104.

10. Welborn, T.A., et al., Serum-insulin in essential hypertension and in peripheral vascular disease. Lancet, 1966. 1(7451): p. 1336-7.

11. Ferrannini, E., et al., Insulin resistance in essential hypertension. N Engl J Med, 1987. 317(6): p. 350-7. 
12. Ferrannini, E., et al., Insulin resistance, hyperinsulinemia, and blood pressure: role of age and obesity. European Group for the Study of Insulin Resistance (EGIR). Hypertension, 1997. 30(5): p. $1144-9$.

13. Shen, S.W., G.M. Reaven, and J.W. Farquhar, Comparison of impedance to insulin-mediated glucose uptake in normal subjects and in subjects with latent diabetes. J Clin Invest, 1970. 49(12): p. 215160.


with fasting hyperglycemia. J Clin Invest, 1975. 55(3): p. 454-61.

15. Olefsky, J., J.W. Farquhar, and G. Reaven, Relationship between fasting plasma insulin level and resistance to insulin-mediated glucose uptake in normal and diabetic subjects. Diabetes, 1973. 22(7): p. 507-13.

16. Fuh, M.M., et al., Abnormalities of carbohydrate and lipid metabolism in patients with hypertension. Arch Intern Med, 1987. 147(6): p. 1035-8.

17. Zavaroni, I., et al., Association between salt sensitivity and insulin concentrations in patients with hypertension. Am J Hypertens, 1995. 8(8): p. 855-8.

18. Reaven, G.M., H. Lithell, and L. Landsberg, Hypertension and associated metabolic abnormalities--the role of insulin resistance and the sympathoadrenal system. N Engl J Med, 1996. 334(6): p. $374-81$.

19. Abbasi, F., et al., Comparison of plasminogen activator inhibitor-1 concentration in insulin-resistant versus insulin-sensitive healthy women. Arterioscler Thromb Vasc Biol, 1999. 19(11): p. 2818-21.

20. Facchini, F., et al., Relationship between resistance to insulin-mediated glucose uptake, urinary uric acid clearance, and plasma uric acid concentration. JAMA, 1991. 266(21): p. 3008-11.

21. Reaven, G.M., et al., Role of insulin in endogenous hypertriglyceridemia. J Clin Invest, 1967. 46(11): p. 1756-67.

22. Golay, A., et al., High density lipoprotein (HDL) metabolism in noninsulin-dependent diabetes mellitus: measurement of HDL turnover using tritiated HDL. J Clin Endocrinol Metab, 1987. 65(3): p. 512-8.

23. Reaven, G.M., et al., Insulin resistance and hyperinsulinemia in individuals with small, dense low density lipoprotein particles. J Clin Invest, 1993. 92(1): p. 141-6.

24. Devereux, R.B., et al., Impact of diabetes on cardiac structure and function: the strong heart study. Circulation, 2000. 101(19): p. 2271-6.

25. Ilercil, A., et al., Relationship of impaired glucose tolerance to left ventricular structure and function: The Strong Heart Study. Am Heart J, 2001. 141(6): p. 992-8.

26. Levy, D., et al., Prognostic implications of echocardiographically determined left ventricular mass in the Framingham Heart Study. N Engl J Med, 1990. 322(22): p. 1561-6.

27. Ghali, J.K., et al., The prognostic role of left ventricular hypertrophy in patients with or without coronary artery disease. Ann Intern Med, 1992. 117(10): p. 831-6.

28. Lorell, B.H. and B.A. Carabello, Left ventricular hypertrophy: pathogenesis, detection, and prognosis. Circulation, 2000. 102(4): p. 470-9. 
29. Harshfield, G.A., et al., Genetic and environmental influences on echocardiographically determined left ventricular mass in black twins. Am J Hypertens, 1990. 3(7): p. 538-43.

30. Chien, K.L., et al., Heritability and major gene effects on left ventricular mass in the Chinese population: a family study. BMC Cardiovasc Disord, 2006. 6: p. 37.

31. Sharma, P., et al., Heritability of left ventricular mass in a large cohort of twins. J Hypertens, 2006. 24(2): p. 321-4.

32. Bombelli, M., et al., Left ventricular hypertrophy increases cardiovascular risk independently of inoffice and out-of-office blood pressure values. J Hypertens, 2009. 27(12): p. 2458-64.

33. Levy, D., et al., Left ventricular mass and incidence of coronary heart disease in an elderly cohort. The Framingham Heart Study. Ann Intern Med, 1989. 110(2): p. 101-7.

34. Koren, M.J., et al., Relation of left ventricular mass and geometry to morbidity and mortality in uncomplicated essential hypertension. Ann Intern Med, 1991. 114(5): p. 345-52.

35. Aronow, W.S., M. Koenigsberg, and K.S. Schwartz, Usefulness of echocardiographic left ventricular hypertrophy in predicting new coronary events and atherothrombotic brain infarction in patients over 62 years of age. Am J Cardiol, 1988. 61(13): p. 1130-2.

36. Gardin, J.M., et al., M-mode echocardiographic predictors of six- to seven-year incidence of coronary heart disease, stroke, congestive heart failure, and mortality in an elderly cohort (the Cardiovascular Health Study). Am J Cardiol, 2001. 87(9): p. 1051-7.

37. Desai, C.S., et al., Usefulness of Left Ventricular Mass and Geometry for Determining 10-Year Prediction of Cardiovascular Disease in Adults Aged $>65$ Years (from the Cardiovascular Health Study). Am J Cardiol, 2016. 118(5): p. 684-90.

38. Du Bois, D. and E.F. Du Bois, A formula to estimate the approximate surface area if height and weight be known. 1916. Nutrition, 1989. 5(5): p. 303-11; discussion 312-3.

39. Lang, R.M., et al., Recommendations for cardiac chamber quantification by echocardiography in adults: an update from the American Society of Echocardiography and the European Association of Cardiovascular Imaging. Eur Heart J Cardiovasc Imaging, 2015. 16(3): p. 233-70.

40. Nagueh, S.F., et al., Recommendations for the Evaluation of Left Ventricular Diastolic Function by Echocardiography: An Update from the American Society of Echocardiography and the European Association of Cardiovascular Imaging. J Am Soc Echocardiogr, 2016. 29(4): p. 277-314.

41. Chau, J.Y., et al., Daily sitting time and all-cause mortality: a meta-analysis. PLoS One, 2013. 8(11): p. e80000.

42. Ryan, H., A. Trosclair, and J. Gfroerer, Adult current smoking: differences in definitions and prevalence estimates-NHIS and NSDUH, 2008. J Environ Public Health, 2012. 2012: p. 918368-78.

43. Moos, R.H., et al., Older adults' alcohol consumption and late-life drinking problems: a 20-year perspective. Addiction, 2009. 104(8): p. 1293-302.

44. Collaborators, G.B.D.O., et al., Health Effects of Overweight and Obesity in 195 Countries over 25 Years. N Engl J Med, 2017. 377(1): 13-27. 
45. Whelton, P.K., et al., 2017 ACC/AHA/AAPA/ABC/ACPM/AGS/APhA/ASH/ASPC/NMA/PCNA Guideline for the Prevention, Detection, Evaluation, and Management of High Blood Pressure in Adults: A Report of the American College of Cardiology/American Heart Association Task Force on Clinical Practice Guidelines. Hypertension, 2018. 71(6): p. e13-e115.

46. d'Emden, M.C., et al., Guidance concerning the use of glycated haemoglobin (HbA1c) for the diagnosis of diabetes mellitus. Med J Aust, 2015. 203(2): p. 89-90.

47. Kim, S.H., et al., Association of insulin resistance and coronary artery remodeling: an intravascular ultrasound study. Cardiovasc Diabetol, 2015. 14: p. 74.

48. Wu, L. and K.G. Parhofer, Diabetic dyslipidemia. Metabolism, 2014. 63(12): p. 1469-79.

49. Elshazly, M.B., et al., Implications of Total to High-Density Lipoprotein Cholesterol Ratio Discordance With Alternative Lipid Parameters for Coronary Atheroma Progression and Cardiovascular Events. Am J Cardiol, 2016. 118(5): p. 647-55.

50. Kerola, T., et al., Long-term prognostic impact of hyperuricemia in community. Scand J Clin Lab Invest, 2019. 79(3): p. 148-153.

51. Oktay, A.A., et al., Current Perspectives on Left Ventricular Geometry in Systemic Hypertension. Prog Cardiovasc Dis, 2016. 59(3): p. 235-246.

52. Silbiger, J.J., Pathophysiology and Echocardiographic Diagnosis of Left Ventricular Diastolic Dysfunction. J Am Soc Echocardiogr, 2019. 32(2): p. 216-232 e2.

53. Galderisi, M., et al., Standardization of adult transthoracic echocardiography reporting in agreement with recent chamber quantification, diastolic function, and heart valve disease recommendations: an expert consensus document of the European Association of Cardiovascular Imaging. Eur Heart $\mathrm{J}$ Cardiovasc Imaging, 2017. 18(12): p. 1301-1310.

54. Nagueh, S.F., Left Ventricular Diastolic Function: Understanding Pathophysiology, Diagnosis, and Prognosis With Echocardiography. JACC Cardiovasc Imaging, 2020. 13(1 Pt 2): p. 228-244.

55. Costa, C.H., et al., Serum insulin levels, 24-hour blood pressure profile, and left ventricular mass in nonobese hypertensive patients. Hypertension, 1995. 26(6 Pt 2): p. 1085-8.

56. Galvan, A.Q., et al., Insulin resistance and hyperinsulinemia: No independent relation to left ventricular mass in humans. Circulation, 2000. 102(18): p. 2233-8.

57. Singh, B. and A. Saxena, Surrogate markers of insulin resistance: A review. World J Diabetes, 2010. $1(2)$ : p. 36-47.

58. Bonora, E., et al., Homeostasis model assessment closely mirrors the glucose clamp technique in the assessment of insulin sensitivity: studies in subjects with various degrees of glucose tolerance and insulin sensitivity. Diabetes Care, 2000. 23(1): p. 57-63.

59. Sasson, Z., et al., Insulin resistance is an important determinant of left ventricular mass in the obese. Circulation, 1993. 88(4 Pt 1): p. 1431-6.

60. Lind, L., et al., Left ventricular hypertrophy in hypertension is associated with the insulin resistance metabolic syndrome. J Hypertens, 1995. 13(4): p. 433-8. 
61. Cauwenberghs, N., et al., Relation of Insulin Resistance to Longitudinal Changes in Left Ventricular Structure and Function in a General Population. J Am Heart Assoc, 2018. 7(7).

62. Nwabuo, C.C. and R.S. Vasan, Pathophysiology of Hypertensive Heart Disease: Beyond Left Ventricular Hypertrophy. Curr Hypertens Rep, 2020. 22(2): p. 11.

63. Sato, A., et al., Left ventricular hypertrophy in normoalbuminuric type 2 diabetic patients not taking antihypertensive treatment. QJM, 2005. 98(12): p. 879-84.

64. Santra, S., et al., Comparison of left ventricular mass in normotensive type 2 diabetes mellitus patients with that in the nondiabetic population. J Cardiovasc Dis Res, 2011. 2(1): p. 50-6.

65. Velagaleti, R.S., et al., Relations of insulin resistance and glycemic abnormalities to cardiovascular magnetic resonance measures of cardiac structure and function: the Framingham Heart Study. Circ Cardiovasc Imaging, 2010. 3(3): p. 257-63.

66. Yilmaz, S., et al., Diabetic Cardiomyopathy; Summary of 41 Years. Korean Circ J, 2015. 45(4): p. 26672.

67. Letonja, M. and D. Petrovic, Is diabetic cardiomyopathy a specific entity? World J Cardiol, 2014. 6(1): p. 8-13.

68. Lepira, F.B., et al., Clinical correlates of left ventricular hypertrophy in black patients with arterial hypertension. Cardiovasc J S Afr, 2006. 17(1): p. 7-11.

69. Devereux, R.B., et al., Relation of insulin to left ventricular geometry and function in African American and white hypertensive adults: the HyperGEN study. Am J Hypertens, 2002. 15(12): p. 1029-35.

70. Olszanecka, A., et al., Relationships of insulin-like growth factor-1, its binding proteins, and cardiometabolic risk in hypertensive perimenopausal women. Metabolism, 2017. 69: p. 96-106.

71. Lopez, B., A. Gonzalez, and J. Diez, Circulating biomarkers of collagen metabolism in cardiac diseases. Circulation, 2010. 121(14): p. 1645-54.

72. Ihm, S.H., et al., Serum carboxy-terminal propeptide of type I procollagen (PIP) is a marker of diastolic dysfunction in patients with early type 2 diabetes mellitus. Int J Cardiol, 2007. 122(3): p. e36-8.

73. Wachtell, K., et al., Left ventricular filling patterns in patients with systemic hypertension and left ventricular hypertrophy (the LIFE study). Losartan Intervention For Endpoint. Am J Cardiol, 2000. 85(4): p. 466-72.

74. Spinale, F.G., Bioactive peptide signaling within the myocardial interstitium and the matrix metalloproteinases. Circ Res, 2002. 91(12): p. 1082-4.

75. Lahera, V., et al., Role of Mitochondrial Dysfunction in Hypertension and Obesity. Curr Hypertens Rep, 2017. 19(2): p. 11.

76. Laviades, C., G. Mayor, and J. Diez, [The presence of diastolic dysfunction in hypertensive patients without left ventricular hypertrophy]. Med Clin (Barc), 1991. 97(5): p. 166-9.

77. Solomon, S.D., et al., Effect of the direct Renin inhibitor aliskiren, the Angiotensin receptor blocker losartan, or both on left ventricular mass in patients with hypertension and left ventricular hypertrophy. Circulation, 2009. 119(4): p. 530-7. 
78. Wachtell, K., et al., Change in diastolic left ventricular filling after one year of antihypertensive treatment: The Losartan Intervention For Endpoint Reduction in Hypertension (LIFE) Study. Circulation, 2002. 105(9): p. 1071-6.

79. Gibbs, B.B., et al., Sedentary screen time and left ventricular structure and function: the CARDIA study. Med Sci Sports Exerc, 2014. 46(2): p. 276-83.

80. Annis C., P.B., Sidibe Moussa, The Influence of Ethnicity in the Relationship between Sedentary Screen Time and Left Ventricular Mass: Insights from the MAG-SALVAGES. WJCD, 2017. 7: p. 13.

81. Fox, E., et al., Body mass index and blood pressure influences on left ventricular mass and geometry in African Americans: The Atherosclerotic Risk In Communities (ARIC) Study. Hypertension, 2004. 44(1): p. 55-60.

82. Avelar, E., et al., Left ventricular hypertrophy in severe obesity: interactions among blood pressure, nocturnal hypoxemia, and body mass. Hypertension, 2007. 49(1): p. 34-9.

83. Woodiwiss, A.J., et al., Obesity promotes left ventricular concentric rather than eccentric geometric remodeling and hypertrophy independent of blood pressure. Am J Hypertens, 2008. 21(10): p. 114451.

84. Grossman, W. and W.J. Paulus, Myocardial stress and hypertrophy: a complex interface between biophysics and cardiac remodeling. J Clin Invest, 2013. 123(9): p. 3701-3.

85. Abel, E.D., K.M. O'Shea, and R. Ramasamy, Insulin resistance: metabolic mechanisms and consequences in the heart. Arterioscler Thromb Vasc Biol, 2012. 32(9): p. 2068-76.

86. George, J. and A.D. Struthers, The role of urate and xanthine oxidase inhibitors in cardiovascular disease. Cardiovasc Ther, 2008. 26(1): p. 59-64.

87. Kurata, A., Y. Shigematsu, and J. Higaki, Sex-related differences in relations of uric acid to left ventricular hypertrophy and remodeling in Japanese hypertensive patients. Hypertens Res, 2005. 28(2): p. 133-9.

88. Matsumura, K., et al., Gender-related association of serum uric acid and left ventricular hypertrophy in hypertension. Circ J, 2006. 70(7): p. 885-8.

89. Xaplanteris, P., et al., Uric acid levels, left ventricular mass and geometry in newly diagnosed, never treated hypertension. J Hum Hypertens, 2011. 25(5): p. 340-2.

90. Catena, C., et al., Uricaemia and left ventricular mass in hypertensive patients. Eur J Clin Invest, 2014. 44(10): p. 972-81.

91. Cuspidi, C., et al., Uric Acid and New Onset Left Ventricular Hypertrophy: Findings From the PAMELA Population. Am J Hypertens, 2017. 30(3): p. 279-285.

92. Gingles, C.R., et al., Allopurinol treatment adversely impacts left ventricular mass regression in patients with well-controlled hypertension. J Hypertens, 2019. 37(12): p. 2481-2489.

93. Szwejkowski, B.R., et al., Allopurinol reduces left ventricular mass in patients with type 2 diabetes and left ventricular hypertrophy. J Am Coll Cardiol, 2013. 62(24): p. 2284-93. 
94. Takimoto, E. and D.A. Kass, Role of oxidative stress in cardiac hypertrophy and remodeling. Hypertension, 2007. 49(2): p. 241-8.

95. Takimoto, E., et al., Oxidant stress from nitric oxide synthase-3 uncoupling stimulates cardiac pathologic remodeling from chronic pressure load. J Clin Invest, 2005. 115(5): p. 1221-31.

96. Corry, D.B., et al., Uric acid stimulates vascular smooth muscle cell proliferation and oxidative stress via the vascular renin-angiotensin system. J Hypertens, 2008. 26(2): p. 269-75.

97. Yu, M.A., et al., Oxidative stress with an activation of the renin-angiotensin system in human vascular endothelial cells as a novel mechanism of uric acid-induced endothelial dysfunction. J Hypertens, 2010. 28(6): p. 1234-42.

98. Cheng, T.H., et al., Uric acid activates extracellular signal-regulated kinases and thereafter endothelin1 expression in rat cardiac fibroblasts. Int J Cardiol, 2010. 139(1): p. 42-9.

99. Ramirez-Sandoval, J.C., L.G. Sanchez-Lozada, and M. Madero, Uric Acid, Vascular Stiffness, and Chronic Kidney Disease: Is There a Link? Blood Purif, 2017. 43(1-3): p. 189-195.

100. Brown, D.W., W.H. Giles, and J.B. Croft, Left ventricular hypertrophy as a predictor of coronary heart disease mortality and the effect of hypertension. Am Heart J, 2000. 140(6): p. 848-56.

101. Cuspidi, C., et al., Prevalence of left-ventricular hypertrophy in hypertension: an updated review of echocardiographic studies. J Hum Hypertens, 2012. 26(6): p. 343-9.

\section{Tables}

Table1 General characteristics of black hypertensive patients at CMK hopital stratified by the presence or absence of left ventricular hypertrophy, January to December 2019 


\begin{tabular}{|c|c|c|c|}
\hline \multirow[t]{2}{*}{ Characteristics } & LVH+ & LVH- & \multirow[t]{2}{*}{$\mathrm{p}$ value } \\
\hline & $n=88$ & $n=132$ & \\
\hline \multicolumn{4}{|c|}{ Demographic characteristics } \\
\hline Age (years) & $52.6 \pm 10.6$ & $50.3 \pm 9.5$ & 0.096 \\
\hline Gender & & & 0.421 \\
\hline Male & $52(59.1)$ & $81(61.4)$ & \\
\hline Female & $36(40.9)$ & $51(38.6)$ & \\
\hline \multicolumn{4}{|c|}{ Medical \& social history } \\
\hline Known HTN & $60(68.2)$ & $76(57.6)$ & 0.074 \\
\hline ND HTN & $28(31.8)$ & $56(42.4)$ & 0.149 \\
\hline Cigaret smoking & $87(98.9)$ & 132(100.0) & 0.400 \\
\hline Alcohol intake & $85(96.6)$ & $128(97.0)$ & 0.582 \\
\hline Menopausal & 14(38.9) & $27(52.9)$ & 0.141 \\
\hline \multicolumn{4}{|c|}{ Anthopomorphic measurments } \\
\hline $\mathrm{BMI}\left(\mathrm{Kg} / \mathrm{m}^{2}\right)$ & $32.6 \pm 5.1$ & $28.7 \pm 4.3$ & $<0.001$ \\
\hline WC $(\mathrm{Cm})$ & $109.3 \pm 13.2$ & $99.3 \pm 10.0$ & $<0.001$ \\
\hline $\mathrm{HC}(\mathrm{Cm})$ & $112.7 \pm 9.9$ & $103.8 \pm 9.2$ & $<0.001$ \\
\hline Overweight & $22(25.0)$ & $64(48.5)$ & $<0.001$ \\
\hline Total obesity & $65(73.9)$ & $47(35.6)$ & $<0.001$ \\
\hline abdominal Obesity & $34(61.4)$ & $43(32.6)$ & $<0.001$ \\
\hline \multicolumn{4}{|l|}{ Lifestyle history } \\
\hline Sedentarity & $71(80.7)$ & $52(39.4)$ & $<0.001$ \\
\hline \multicolumn{4}{|c|}{ Treatment history \& examination findings } \\
\hline Uncontrolled HTN & $20(22,7)$ & $18(13,6)$ & 0,060 \\
\hline $\mathrm{SBP}(\mathrm{mmHg})$ & $138,8 \pm 7,8$ & $133,4 \pm 7,2$ & 0,048 \\
\hline $\mathrm{DBP}(\mathrm{mmHg})$ & $82,5 \pm 8,7$ & $79,9 \pm 9,1$ & 0,087 \\
\hline HR (bpm) & $62.1 \pm 13.5$ & $70.0 \pm 13.4$ & 0.199 \\
\hline
\end{tabular}

HTN=hypertension ; ND HTN= newly-diagnosed hypertension ; WC = Waist circumference ; $\mathrm{BMI}=$ Body mass index ; $\mathrm{HC}=$ hip circumference ; HR=Heart rate ; $\mathrm{SBP}=$ Systolic Blood Pressure ; $\mathrm{DBP}=$ Diastolic Blood 
Pressure.

Table 2 Echographic and biologic characteristics of black hypertensive patients at CMK hospital stratified by the presence or absence of left ventricular hypertrophy, January to December 2019 


\begin{tabular}{|c|c|c|c|}
\hline Variables & $\begin{array}{l}\text { LVH+ } \\
n=88\end{array}$ & $\begin{array}{l}\text { LVH- } \\
n=132\end{array}$ & $\mathbf{P}$ \\
\hline \multicolumn{4}{|c|}{ Echocardiographic measurements } \\
\hline LVED (mm) & $46.5 \pm 4.4$ & $42.9 \pm 4,1$ & $<0.001$ \\
\hline IVS (mm) & $12.7 \pm 1.1$ & $10.7 \pm 1.5$ & $<0.001$ \\
\hline PWT (mm) & $12.5 \pm 0.8$ & $10.7 \pm 1.5$ & $<0.001$ \\
\hline SWT (mm) & $25.2 \pm 1.6$ & $21.3 \pm 2.9$ & $<0.001$ \\
\hline $\operatorname{LVEF}(\%)$ & $63.8 \pm 5.4$ & $65.1 \pm 4.9$ & 0.062 \\
\hline LVM (g) & $222.2 \pm 38.4$ & $156.8 \pm 34.8$ & $<0.001$ \\
\hline $\operatorname{LVMIh}\left(\mathrm{g} / \mathrm{m}^{2,7}\right)$ & $54.7 \pm 8.4$ & $37.6 \pm 6.6$ & $<0.001$ \\
\hline LVMIbs $\left(\mathrm{g} / \mathrm{m}^{2}\right)$ & $108.5 \pm 15.7$ & $79.7 \pm 15.0$ & $<0.001$ \\
\hline RWT & $0.55 \pm 0.1$ & $0.50 \pm 0.1$ & 0.001 \\
\hline $\mathrm{E}(\mathrm{Cm} / \mathrm{s})$ & $0.85 \pm 0.6$ & $1.08 \pm 0.6$ & 0.029 \\
\hline E/A ratio & $0.71 \pm 0.2$ & $0.99 \pm 0.2$ & 0.034 \\
\hline DT (ms) & $215.8 \pm 39.4$ & $172.8 \pm 37.7$ & $<0.001$ \\
\hline LAA $\left(\mathrm{Cm}^{2}\right)$ & $17.3 \pm 3.5$ & $14.7 \pm 2.7$ & $凶 0.001$ \\
\hline SPAP $(\mathrm{mmHg})$ & $26.9 \pm 3.1$ & $26.0 \pm 2.7$ & 0.019 \\
\hline \multicolumn{4}{|l|}{ Biologic parameters } \\
\hline TC (mmolL) & $5.5 \pm 1.0$ & $5.4 \pm 1.0$ & 0.305 \\
\hline LDLc (mmol/L) & $3.8 \pm 1.1$ & $3.6 \pm 1.1$ & 0.126 \\
\hline Triglycrides (mmol/L) & $1.25 \pm 0.6$ & $1.05 \pm 0.6$ & 0.027 \\
\hline $\mathrm{HDLc}(\mathrm{mmol} / \mathrm{L})$ & $1.1 \pm 0.3$ & $1.3 \pm 0.4$ & 0.003 \\
\hline Glycemia (mmol/L) & $6.3 \pm 2.1$ & $5.4 \pm 1.6$ & $<0.001$ \\
\hline HbA1C (\%) & $6.3 \pm 1.6$ & $5.9 \pm 1.1$ & 0.016 \\
\hline Insulinemia (mmol/L) & $122.8 \pm 43.1$ & $72.7 \pm 25.8$ & $<0.001$ \\
\hline Al & $5.2 \pm 1.6$ & $4.6 \pm 1.8$ & 0.008 \\
\hline HOMAIR & $2.36 \pm 0.8$ & $1.41 \pm 0.6$ & 0.014 \\
\hline Uric acid (mmolL) & $388.3 \pm 98.4$ & $352.9 \pm 89.5$ & 0.007 \\
\hline Creatinine (mol/L) & $84.7 \pm 22.6$ & $84.3 \pm 16.2$ & 0.854 \\
\hline
\end{tabular}




\begin{tabular}{|llll|}
\hline Calcium (mmol/L) & $2.30 \pm 0.2$ & $2.3 \pm 0.2$ & 0.105 \\
\hline Ionized calcium (mmolL) & $1.20 \pm 0.11$ & $1.22 \pm 0.2$ & 0.331 \\
\hline Phosphore (mmolL) & $1.06 \pm 0.2$ & $1.09 \pm 0.3$ & 0.333 \\
\hline Dyslipidémia & $75(85.2)$ & $98(74.2)$ & 0.036 \\
\hline High Al & $45(51.1)$ & $48(36.4)$ & 0.021 \\
\hline T2DM & $20(22.7)$ & $23(17.4)$ & 0.212 \\
\hline Hyperinsulinisme & $8(9.1)$ & $11(8.3)$ & 0.514 \\
\hline IR & $42(47.7)$ & $2(1.5)$ & $<0.001$ \\
\hline Hyperuricemia & $29(33.0)$ & $22(16.7)$ & 0.004 \\
\hline
\end{tabular}

LVED= Left ventricular end-diastolic diameter ;IVS= interventricular septal thickness; PWT= Posterior wall thickness; SWT = Sum of wall thickness; LVEF= Left ventricular ejection fraction; LVM= left ventricular mass; LVMIh= left ventricular mass indexed to height ${ }^{2,7} ;$ LVMbs= left ventricula mass indexed to body surface area ; RWT=relative wall thickness; $E=$ mitral $E$ wave; $E / A=$ ratio of peak early and late diastolic flow velocities ; $D T=$ deceleration time; $L A A=$ left atrium area; $S P A P=$ systolic pulmonary arterial pressure; $\mathrm{TC}=$ total cholesterol; $\mathrm{LDLC}=$ low density lipoprotein; $L D L-C=$ low density lipoprotein ; HDLC= high density lopoprotein; $\mathrm{Al}=$ atherogenicity index; $\mathrm{HbA1C}=$ glycated haemoglobin; $\mathrm{T} 2 \mathrm{DM}=\mathrm{vtype} 2$ diabetes melitus; HOMAIR= Homeostatic Model Assessment for Insulin Resistance; IR= insulin resistance.

Table 3 Simple linear regression showing determinants of left vetnricular mass (LVM) in 220 black patients with hypertension at CMK hospital, January to December 2019

\begin{tabular}{|llll|}
\hline Variables & $\mathbf{r}$ & $\boldsymbol{\beta}$ & $\mathbf{p}$ \\
\hline Age in years & 0.190 & 0.22 & $\mathbf{0 . 0 0 5}$ \\
\hline HTN duration in years & 0.313 & 0.57 & $<\mathbf{0 . 0 0 1}$ \\
\hline BMI $\left(\mathrm{kg} / \mathrm{m}^{2}\right)$ & 0.444 & 0.99 & $<\mathbf{0 . 0 0 1}$ \\
\hline WC in cm & 0.425 & 0.39 & $<\mathbf{0 . 0 0 1}$ \\
\hline Glycemia $(\mathrm{mmol} / \mathrm{L})$ & 0.201 & 1.19 & $\mathbf{0 . 0 0 3}$ \\
\hline Insuline $(\mathrm{mmol} / \mathrm{L})$ & 0.448 & 0.12 & $<\mathbf{0 . 0 0 1}$ \\
\hline HOMAIR & 0.437 & 5.80 & $<\mathbf{0 . 0 0 1}$ \\
\hline
\end{tabular}

HTN=hypertension ; BMl=body mass index ; WC= waist circumference ; HOMAIR= Homeostatic Model Assessment for Insulin Resistance. 
Table 4 Multiple linear regression showing determinants of left vetnricular mass (LVM) in 220 black patients with hypertension at CMK hospital, January to December 2019

\begin{tabular}{|c|c|c|c|}
\hline \multirow[t]{2}{*}{ Variables } & \multicolumn{3}{|c|}{ LVM Ih } \\
\hline & $\beta$ & SE & p \\
\hline (Constant) & 6.84 & 8.72 & 0.435 \\
\hline Age (years) & 0.14 & 0.104 & 0.183 \\
\hline HTN duration & 0.56 & 0.14 & $<0.001$ \\
\hline BMI $\left(\mathrm{Kg} / \mathrm{m}^{2}\right)$ & 0.67 & 0.23 & 0.004 \\
\hline WC (cm) & 0.001 & 0.09 & 0.994 \\
\hline Glycemia (mmol/L) & 0.06 & 0.46 & 0.903 \\
\hline Insuline (mmolL) & 0.08 & 0.04 & 0.034 \\
\hline HOMAIR & 0.27 & 1.81 & 0.021 \\
\hline$R^{2}=0.683$, overall $p$ & 0.001 & & \\
\hline
\end{tabular}

$\mathrm{HTN}=$ hypertension $; \mathrm{BMI}=$ body mass index $; \mathrm{WC}=$ waist circumference $;$ HOMAIR= Homeostatic Model Assessment for Insulin Resistance

$Y=0.56 X_{1}+0.67 X_{2}+0.08 X_{3}+0.27 X_{4}+6.84$

With $Y=$ LVMIh $; X_{1}=$ HTN duration $; X_{2}=B M I ; X_{3}=$ Insuline and $X_{4}=$ HOMAIR

Table 5 Logistic regression analysis showing determinants of LVH among 220 black hypertensives from CMK hospital, January to December 2019 


\begin{tabular}{|c|c|c|c|c|}
\hline \multirow[t]{2}{*}{ Variables } & \multicolumn{2}{|c|}{ Univariate analysis } & \multicolumn{2}{|c|}{ Multivariate analysis } \\
\hline & $\mathrm{p}$ & OR (95\% Cl) & $\mathrm{p}$ & aOR $(95 \% \mathrm{Cl})$ \\
\hline \multicolumn{5}{|c|}{ Total Obesity } \\
\hline No & & 1 & & 1 \\
\hline Yes & 0.000 & $5.1(2.8-9.3)$ & 0.038 & $2.8(1.06-7.4)$ \\
\hline \multicolumn{5}{|c|}{ Abdominal Obesity } \\
\hline No & & 1 & & 1 \\
\hline Yes & 0.000 & $3.3(1.9-5.8)$ & 0.275 & $1.9(0.6-6.3)$ \\
\hline \multicolumn{5}{|l|}{ Sedentary } \\
\hline No & & 1 & & 1 \\
\hline Yes & 0.000 & $6.4(3.4-12.1)$ & 0.123 & $1.9(0.8-4.5)$ \\
\hline \multicolumn{5}{|l|}{ High Al } \\
\hline No & & 1 & & 1 \\
\hline Yes & 0.031 & $1.8(1.06-3.2)$ & 0.579 & $1.3(0.6-2.9)$ \\
\hline \multicolumn{5}{|c|}{ Hyperuricemia } \\
\hline No & & 1 & & 1 \\
\hline Yes & 0.006 & $2.5(1.3-4.7)$ & 0.145 & $2.1(0.8-5.4)$ \\
\hline \multicolumn{5}{|l|}{ IR } \\
\hline No & & 1 & & 1 \\
\hline Yes & 0.000 & $9.3(3.8-25.5)$ & 0.000 & $8.4(3.7-15.7)$ \\
\hline
\end{tabular}

$\mathrm{Al}=$ atherogenic index $; \mathrm{IR}=$ insulin resistance

Figures 


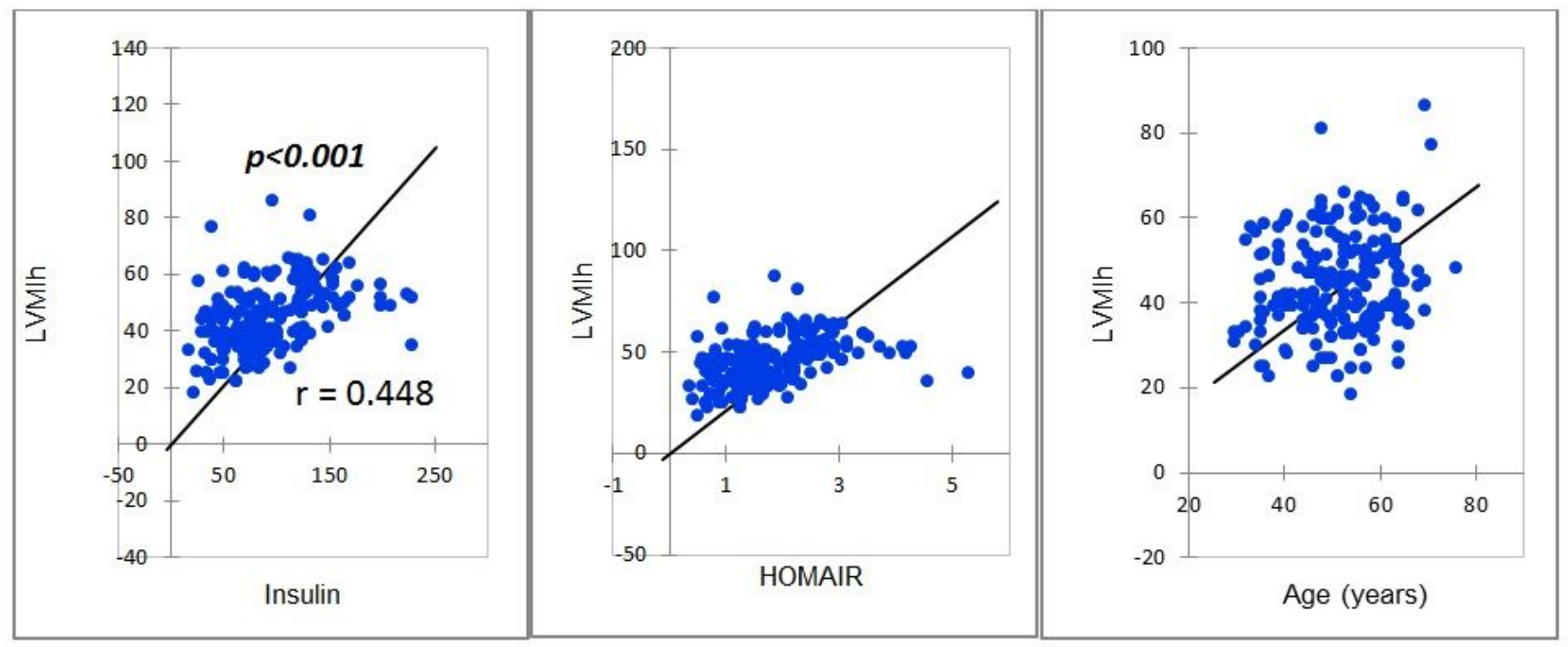

Figure 1A. Explanatory factors for the increase in LVM in simple linear regression.


Figure 1B. Explanatory factors for the increase in LVM in simple linear regression

Figure 1

Figure 1A. Explanatory factors for the increase in LVM in simple linear regression. Figure 1B. Explanatory factors for the increase in LVM in simple linear regression 